\title{
Teleconsulta no SUS durante a pandemia da COVID-19 no Brasil
}

\author{
Teleconsultation at SUS during the COVID-19 pandemic in Brazil \\ Teleconsulta en el SUS durante la pandemia de COVID-19 em Brasil
}

Recebido: 29/06/2021 | Revisado: 06/07/2021 | Aceito: 07/07/2021 | Publicado: 17/07/2021

\author{
Cláudia Batista Mélo \\ ORCID: https://orcid.org/0000-0001-5300-3510 \\ Universidade Federal da Paraíba, Brasil \\ E-mail: claudia.melo@academico.ufpb.br \\ Gabrieli Duarte Farias \\ ORCID: https://orcid.org/0000-0001-6904-2856 \\ Universidade Federal da Paraíba, Brasil \\ E-mail: gabrieli.duarte@academico.ufpb.br \\ Heitor Vinicius Brasileiro Ramalho \\ ORCID: https://orcid.org/0000-0002-4683-0451 \\ Universidade Federal da Paraíba, Brasil \\ E-mail: Heitor.vinicius@academico.ufpb.br \\ Jeann Mateus Gonzaga dos Santos \\ ORCID: https://orcid.org/0000-0001-5663-7076 \\ Universidade Federal da Paraíba, Brasil \\ E-mail: jeann.gonzaga@ academico.ufpb.br \\ Tiago Tavares da Rocha \\ ORCID: https://orcid.org/0000-0003-2086-3551 \\ Universidade Federal da Paraíba, Brasil \\ E-mail: tiago.rocha@academico.ufpb.br \\ Edvaldo José Garcia Gonçalves \\ ORCID: https://orcid.org/0000-0003-2177-9060 \\ Universidade Federal da Paraíba, Brasil \\ E-mail: ejgg@academico.ufpb.br \\ Raquel Bezerra Barbosa de Moura \\ ORCID: https://orcid.org/0000-0002-7490-8754 \\ Universidade Federal da Paraíba, Brasil \\ E-mail: raquel.barbosa@academico.ufpb.br \\ Carmem Silvia Laureano Dalle Piagge \\ ORCID: https://orcid.org/0000-0001-7999-2943 \\ Universidade Federal da Paraíba, Brasil \\ E-mail: carmem.piagge@ academico.ufpb.br
}

\begin{abstract}
Resumo
Devido à pandemia da COVID-19, o Brasil regulamentou a teleconsulta a fim de realizar atendimentos remotos, eletivos e de rotina, evitando o colapso do Sistema Único de Saúde (SUS). O objetivo dessa pesquisa foi investigar a oferta da teleconsulta no SUS e da assistência multiprofissional durante a pandemia nos diversos estados brasileiros. Realizou-se uma revisão da literatura seguido de um estudo documental, descritivo, quantitativo e qualitativo. As coletas dos dados ocorreram a partir de documentos, em domínio público, dispostos em websites oficiais de unidades federativas. Observou-se que $100 \%$ dos estados ofertaram atendimento pré-clínico. No que se refere a disponibilização de teleconsulta para todos os cidadãos, a região Centro-Oeste e Sudeste tiveram 100\% de oferta em seus estados, o Sul $66,7 \%$, o Norte $57,1 \%$ e o Nordeste $44,4 \%$. Foi oferecido teleconsulta com médico em todas as regiões e com cirurgião-dentista apenas na região Nordeste e Sudeste. A disponibilização do programa de Telessaúde ocorreu em $85,2 \%$ dos estados. Conclui-se que a teleconsulta no SUS possibilitou o acesso dos cidadãos aos serviços de saúde de forma remota, principalmente durante a pandemia da COVID-19, e a maioria dos estados brasileiros ofertou o Programa Telessaúde.
\end{abstract}

Palavras-chave: COVID-19; Sistema Único de Saúde; Teleconsulta; Telessaúde.

\begin{abstract}
Due to the Covid-19 pandemic, Brazil regulated the online medical care in order to provide remote attendance, elective and check-ups, avoiding the collapse of the Unified Health System. The purpose of this research was to investigate the offer to online medical care in SUS and multi professional assistence during the pandemic in the various states of the Brazil. It was performed a documentary, descriptive, quantitative and qualitative study. The data collection was made based on documents in public domain, found in officials websites of the states. It was observed that $100 \%$ of the states has offered pre-clinical attendeance. With the regard of the avaiability of the online medical
\end{abstract}


care to all citizens, there was a $100 \%$ reach in the Midwest and the Southwest states, $66 \%$ in the South, $57,1 \%$ in the North and $44,4 \%$ in the Northwest. It was given online medical care with doctors in all regions and with dental surgeon only in the Northwest and Southwest. The affordance of the Telehealth program has occured in $85,2 \%$ of the states. It can be concluded that the teleconsultation in SUS enabled citizens to acess health services remotely, especially during the Covid-19 pandemic, and that the biggest part of the brazilian states has offered the Telehealth Program.

Keywords: COVID-19; Health Unic System; Teleconsultation; Telehealth.

\begin{abstract}
Resumen
Debido a la pandemia del COVID-19, Brasil reguló la teleconsulta para realizar la atención a distancia, electiva y de rutina, evitando el colapso del Sistema Único de Salud (SUS). El objetivo de esta investigación fue investigar la oferta de teleconsulta en el SUS y la asistencia multidisciplinaria durante la pandemia en los diversos estados brasileños. Se realizó una revisión bibliográfica, seguida de un estudio documental, descriptivo, cuantitativo y cualitativo. La recopilación de datos se realizó a partir de documentos de dominio público, disponibles en los sitios $w e b$ oficiales de las unidades federales. Se observó que el $100 \%$ de los estados ofrecían atención preclínica. En cuanto a la disponibilidad de la teleconsulta para todos los ciudadanos, el Medio Oeste y el Sureste tenían una oferta del $100 \%$ en sus estados, el Sur el $66,7 \%$, el Norte el $57,1 \%$ y el Noreste el 44,4\%. La teleconsulta con los médicos se ofreció en todas las regiones y con los dentistas sólo en el noreste y el sureste. La disponibilidad del programa de telesalud se produjo en el $85,2 \%$ de los estados. Se concluye que la teleconsulta en el SUS permitió el acceso de los ciudadanos a los servicios de salud a distancia, especialmente durante la pandemia de COVID-19, y la mayoría de los estados brasileños ofrecieron el Programa de Telesalud.
\end{abstract}

Palabras clave: COVID-19; Sistema Único de Salud; Teleconsulta; Telesalud.

\title{
1. Introdução
}

A pandemia da COVID-19 trouxe desafios anteriormente inimagináveis para o século XXI, em termos de reorganização mundial e as relações entre os indivíduos, desde o convívio com familiares, até o ambiente profissional, mudando, principalmente, a conformidade na rede de saúde e na forma da organização geopolítica em todo o mundo. Em 11 de março de 2020, a Organização Mundial da Saúde (OMS) declarou a Sars-Cov-2, novo coronavírus, uma ameaça global à saúde pública (Nazif-Muñoz, Peña, \& Oulhote, 2021). Posteriormente, no território nacional, no primeiro semestre de 2020, foi instituído o primeiro ato de lockdown por causa do rápido avanço e facilidade de transmissão da COVID-19 (Villela et al., 2021). Com os estudos mais avançados, tornou-se evidente que esse vírus afetava principalmente pessoas idosas e predispostas para adquirir esse vírus, consideradas do grupo de risco (Bergman, Ballin, Nordström, \& Nordström, 2021), posteriormente, descobriu-se que as variantes da COVID-19 afetam também jovens, adultos e pessoas sem comorbidades em maior quantidade, expandindo ainda mais a contaminação. Sabe-se também, que a COVID-19 age atacando o sistema imunológico, desde um simples estágio febril, tosse, calafrios, diarreia, até uma síndrome do desconforto respiratória agudo, insuficiência respiratória e disfunções em múltiplos órgãos por exemplo, onde alcança o estado crítico da doença que ocasiona o maior número de óbitos (O'Keefe, Tong, O'Keefe, \& Tong, 2021).

Os profissionais da saúde também formam um grupo de risco para a COVID-19, por estarem expostos, diariamente, aos pacientes infectados, fazendo com que recebam uma alta carga viral. Além disso, esses profissionais estão submetidos ao enorme estresse dos atendimentos desses pacientes, já que a maioria das localidades não proporciona uma divisão de alas ou hospitais, principalmente em regiões de difícil acesso ou baixa infraestrutura, sendo uma das maiores reclamações desses profissionais: Escassez de Equipamentos de Proteção Individual (EPI), escassez de testes diagnósticos, comprometimento da segurança profissional e o risco do contágio (Caram, Ramos, Almeida, \& Brito, 2021).

Com as recomendações $\mathrm{N}^{\mathrm{o}}$ 036, de 11 de maio de 2020, as medidas de distanciamento social foram adotadas pelo Ministério da Saúde, a fim de conter o avanço progressivo da doença. Tais medidas visam a diminuição da circulação de carros particulares e pessoas, principalmente de trabalhos considerados não essenciais, uso de máscaras obrigatório e medidas sanitárias, como o uso do álcool em gel e dedetização de locais públicos. Contudo, mesmo com restrições, o Brasil não escapou da segunda onda da COVID-19, principalmente após o recuo dos lockdowns estaduais e municipais, o que 
proporcionou um novo aumento nos números de casos da pandemia por causa do distanciamento previamente relaxado em alguns estados brasileiros. Outro grande avanço nas medidas de proteção foi a campanha de vacinação no Brasil, por meio do Sistema Único de Saúde (SUS), da vacina em todo o Brasil, porém, mesmo após a vacinação, continua o alerta dos perigos de contaminação com o novo coronavírus, já que as vacinas estão sendo disponibilizadas em doses separadas para que a ação imunizante atinja uma boa resposta, pois a OMS determinou que apenas as vacinas superiores a $50 \%$ de respostas imunológicas serão aceitáveis (Domingues, 2021).

Visando evitar o colapso no SUS, durante a pandemia, a telemedicina passou a desempenhar papel fundamental na saúde pública, por meio das tecnologias de informação e comunicação (TIC). Essas ferramentas permitem o compartilhamento de informações de modo seguro e rápido, validar e descartar informações, manter a comunicação apesar do distanciamento e, principalmente, realizar atividades de teletrabalho, teleducação, telemedicina, entre outras, além do baixo custo operacional (Ghiglia, 2020). Nesse sentido, o TeleSUS é extremamente necessário no favorecimento do isolamento social, principalmente da parcela populacional com sintomas da COVID-19 ou do grupo de risco. A telessaúde tem como objetivo a expansão e a melhora da rede de serviços de saúde, por meio de ferramentas de tecnologias da informação, que oferecem condições para promover a teleassistência e a teleducação, principalmente da Atenção Primária à Saúde (APS). Outra ferramenta importante é o Consultório Virtual de Saúde da Família, que abrange várias áreas da saúde, não se limitando apenas ao novo coronavírus, dando uma maior autonomia e condição de trabalho para os médicos. Isso permite que a tecnologia facilite as consultas e orientações com médicos, psicólogos, fonoaudiólogos, cirurgiões-dentistas e outros. É importante salientar que a Lei $\mathrm{N}^{\circ} 13.989$, de 15 de abril de 2020, dispõe sobre o uso da telemedicina durante a crise causada pelo novo coronavírus, assegurando o direito de a telemedicina validar prescrições médicas e regulamentando as profissões da área da saúde para que possam ser exercidas de forma virtual (Brasil, 2020a).

Este trabalho se propõe a discutir e investigar a oferta da teleconsulta no SUS e a assistência multiprofissional aos pacientes durante o período de pandemia da COVID-19, avaliando a disponibilização do programa Telessaúde no Brasil.

\section{Metodologia}

Trata-se de um estudo utilizando dois caminhos metodológicos complementares: a revisão bibliográfica e o estudo documental. A princípio foi feita uma revisão abrangente da literatura sobre a oferta da teleconsulta de forma multiprofissional no serviço público durante a pandemia da COVID-19 no Brasil. Foi realizada buscas, no período de 01 de Junho até 09 de Junho de 2021, nas bases bibliográficas MEDLINE, LILACS ScienceDirect e Scielo, usando como descritores e palavras-chave ("COVID-19" Or "Coronavirus" OR "Sars-Cov-2") AND ("Unified Health System" OR "Public service" Or "public") AND ("Teleconsultation" OR "Telehealth" OR "Telemedicine" OR "TeleSUS"). Em seguida, a fim de complementar a pesquisa, realizou-se um estudo observacional, do tipo documental, com procedimento descritivo, quantiqualitativo (Lakatos, Marconi, 2009), discorrendo sobre a oferta de atendimento pré-clínico, de serviço de teleconsulta em saúde e da distribuição e oferta do programa de Telessaúde nos estados brasileiros, durante a pandemia da COVID-19. As pesquisas de natureza documental oportunizam entender o funcionamento e o alcance do objeto pesquisado, através do tratamento analítico dos dados primários (Sá-Silva, Almeida, \& Guindani, 2009). Sendo assim, a relevância dessa pesquisa consiste na obtenção de resultados quantitativos e qualitativos acerca da disponibilidade dos serviços de TeleSUS, Consultório Virtual de Saúde da Família e Telessaúde dentro das unidades federativas, apontando suas prevalências e variações, além de citar outros programas e projetos desenvolvidos por alguns estados brasileiros.

Os recursos documentais utilizados foram sites oficiais de unidades federativas brasileiras, tais quais: o website do Conselho Nacional de Secretarias de Saúde (CONASEMS), em cada estado do país. Além dos websites do Programa de Telessaúde Brasil Redes (https://aps.bvs.br/rede-de-colaboradores/); do TeleSUS (https://aps.saude.gov.br/ape/corona 
/telesus); e do Departamento de Saúde Digital do Ministério da Saúde (DESD-MS; https://saudedigital.saude.gov.br/). Dessa forma, recolheu-se os dados disponíveis para consulta pública, dispensando a submissão ao Comitê de Ética e Pesquisa, tendo em vista a Instrução Normativa $N^{\circ} 510 / 2016$, que regulamenta sobre as normas aplicáveis a pesquisas em Ciências Humanas e Sociais (Brasil, 2016).

Foram selecionados os questionamentos a serem investigados e compilados, possibilitando a categorização dos dados. Dentre as informações obtidas, aferiu-se quais estados dispunham de teleconsultas, assim como, qual era o público alvo que poderia desfrutar desse recurso e quais as restrições para esses serviços. Também foi avaliado o oferecimento dos serviços do Telessaúde e as interfaces disponibilizadas, como: teleconsulta, telediagnóstico, tele-educação e telerrastreamento para profissionais da saúde.

A digitação dos dados e a análise estatística foram realizadas através do programa SPSS (Statistical Package for the Social Sciences), na versão 23.0, sendo analisados por meio de estatística descritiva.

\section{Resultados}

\subsection{Serviços de teleatendimento no Sistema Único de Saúde}

Os principais projetos desenvolvidos pelo SUS para ofertar teleatendimento à sociedade, são: TeleSUS, Consultório Virtual da Saúde da Família e Telessaúde. O TeleSUS constitui-se como uma estratégia para o enfrentamento da COVID-19 funcionando como um serviço de diagnóstico pré-clínico de saúde, oferecendo informações sobre a doença e orientação quanto ao momento ideal de procurar atendimento presencial (Brasil, 2020c). O Consultório Virtual da Saúde surge por meio da parceria entre a Secretaria de Atenção Primária à Saúde (SAPS) com o Hospital Albert Einstein, para que os profissionais da atenção básica ofereçam teleconsultas à população, principalmente pessoas com doenças crônicas, por meio de plataformas virtuais e telefone no período de pandemia (Brasil, 2020b). O Programa Telessaúde Brasil Redes, instituído em 2007 e ampliado em 2011, tem como objetivo o fortalecimento e melhoria no atendimento da atenção básica, utilizando as tecnologias da informação e comunicação (Brasil, 2007).

\subsection{Uso dos serviços de teleatendimento para auxiliar no combate à COVID-19}

No atual estado pandêmico, em decorrência do novo coronavírus, o SUS se voltou para consultas remotas com o objetivo de minimizar o risco de infecção de profissionais da saúde e usuários de vários grupos sociais. Esses aparatos foram de extrema importância e garantiram que grande parte da sociedade brasileira tivesse acesso às consultas e informações referentes à pandemia atual (Almeida, Marabujo, \& Gonçalves, 2021). Essa rápida implantação dos serviços de teleatendimento garantiu uma breve melhora em relação à superlotação dos locais físicos de saúde pública, além de fornecer atendimento primário referentes à COVID-19. Dessa forma, as múltiplas formas de aplicações na saúde permitiram uma comunicação entre profissionais e usuários, que possibilitou a obtenção de diagnósticos, receitas e acompanhamentos clínicos, além de campos como a telemedicina e teleducação, viabilizando o bem-estar social e as necessárias mudanças perante a pandemia no Brasil (Caetano et al., 2020).

\subsection{Oferta dos serviços de teleconsulta durante à COVID-19}

Há diferenças regionais significativas quanto ao uso e implementação da telessaúde/telemedicina, decorrente do fato de que algumas regiões já ofereciam esses serviços antes da pandemia. A necessidade de atendimento especializado em regiões com difícil acesso e desassistidas pela assistência profissional, resultou em parcerias com hospitais e entidades localizadas em outras regiões, garantindo atendimento integral aos seus cidadãos. Essas diferenças passaram a ser evidentes na forma como os estados organizaram seus serviços e na sua variedade de opções. Verificou-se que todas as regiões 
ofereceram atendimento pré-clínico para os habitantes durante o período pandêmico (100\%), mas há uma diferença regional quanto a oferta da teleconsulta direcionada para os cidadãos de acordo com sua apresentação clínica. A região Norte apresentou uma baixa quantidade de ofertas de teleconsultas para pessoas com doenças crônicas $(28,6 \%)$, a região CentroOeste (50\%) apresentou uma oferta mediana, a região Nordeste (55,5\%) teve uma oferta um pouco acima da média, enquanto que a região Sudeste e a região Sul (100\%) foram as que apresentaram uma maior oferta de teleconsultas a esse público, ou seja, todos os estados dessas regiões disponibilizaram teleconsultas direcionadas para pacientes com doenças crônicas.

No que se refere à oferta de teleconsulta para o público em geral, as regiões Centro-Oeste e Sudeste (100\%) tiveram uma oferta máxima de teleconsulta para os seus habitantes, a região Sul (66,7\%) apresentou-se acima da média, logo em seguida vem a região Norte $(57,1 \%)$ e a região Nordeste $(44,4 \%)$ foi a que menos ofertou atendimento para seus habitantes.

Quanto à oferta de teleconsulta para pessoas com sintomas de COVID-19, verificou-se que a região Sudeste (33,3\%) foi a que mais ofertou essa modalidade, seguida pela região Nordeste $(22,2 \%)$ e a região Norte $(14,3 \%)$ foi a que menos ofertou essa modalidade (Figura 1).

Figura 1. Distribuição da oferta dos serviços de teleconsulta em saúde, durante a pandemia da COVID-19 entre as regiões do Brasil.

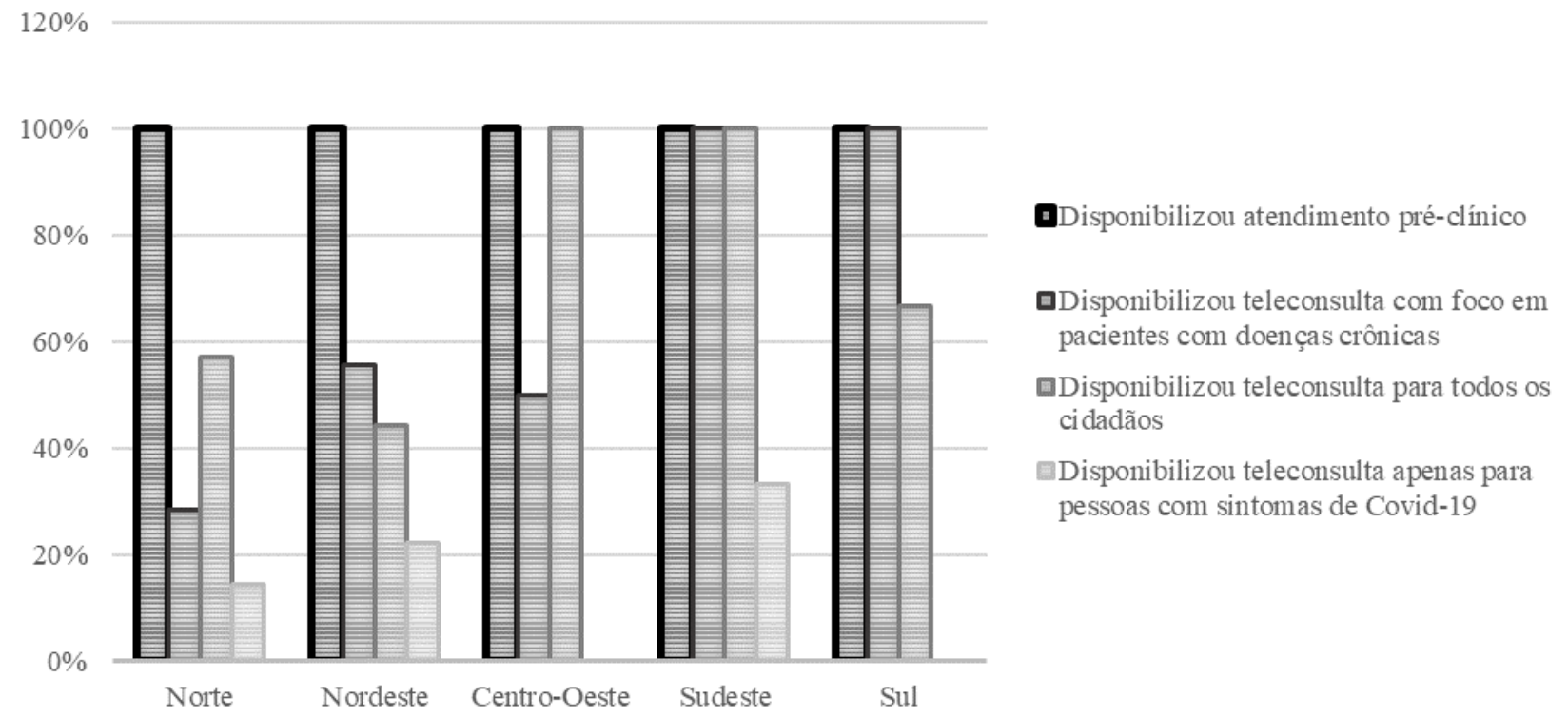

Fonte: Autores.

\subsection{Característica dos serviços de teleconsulta e assistência multiprofissional}

Em relação às características dos serviços de teleatendimento ofertados em cada região do Brasil, pode-se perceber que a região Nordeste foi a que apresentou mais estados com restrição quanto ao teleatendimento. No estado da Bahia, não se indicava teleconsulta para pacientes com sintomas graves que necessitavam de exames físicos ou complementares, no estado de Pernambuco e do Piauí, a teleconsulta era indicada somente para pacientes com sintomas de COVID-19. Quanto ao teleatendimento direcionado aos pacientes com necessidades especiais, apenas a região Centro-Oeste ofereceu essa modalidade, utilizando a Língua Brasileira de Sinais (LIBRAS). Quanto à oferta de teleconsultas com profissionais de saúde, as regiões que ofereceram teleatendimento com cirurgiões-dentistas foram somente a região Nordeste e Sudeste; todas as regiões ofereceram teleconsulta com médicos; e somente a região Sul não ofereceu teleconsulta com enfermeiros. Todas as regiões ofereceram teleconsultas com outros profissionais de saúde, como psicólogos, nutricionistas, fisioterapeutas, fonoaudiólogos, terapeutas ocupacionais e médicos especialistas em gastroenterologia, cardiologia e homeopatia (Quadro 1). 
Quadro 1. Características dos serviços de teleatendimento ofertados em cada região do Brasil, durante a pandemia da COVID-19.

\begin{tabular}{|l|c|c|c|c|c|}
\hline Variáveis & Norte & Nordeste & $\begin{array}{l}\text { Centro- } \\
\text { Oeste }\end{array}$ & Sudeste & Sul \\
\hline Região que apresentou restrição quanto ao teleatendimento & & $\mathrm{X}$ & & & \\
\hline $\begin{array}{l}\text { Região que apresentou plano de teleatendimento } \\
\text { direcionado aos pacientes com necessidades especiais }\end{array}$ & & & $\mathrm{X}$ & & \\
\hline Região que ofertou teleconsulta com cirurgião-dentista & & $\mathrm{X}$ & & $\mathrm{X}$ & \\
\hline Região que ofertou teleconsulta com médico & $\mathrm{X}$ & $\mathrm{X}$ & $\mathrm{X}$ & $\mathrm{X}$ & $\mathrm{X}$ \\
\hline Região que ofertou teleconsulta com enfermeiro & $\mathrm{X}$ & $\mathrm{X}$ & $\mathrm{X}$ & $\mathrm{X}$ & \\
\hline Região que ofertou teleconsulta com outros profissionais & $\mathrm{X}$ & $\mathrm{X}$ & $\mathrm{X}$ & $\mathrm{X}$ & $\mathrm{X}$ \\
\hline
\end{tabular}

Fonte: Autores.

Quanto à emissão de receitas e atestados durante o atendimento por teleconsulta, somente $37 \%$ de todos os estados informaram emitir. Na região Norte, não houve estados que informaram a emissão; na região Nordeste, somente quatro estados emitem, dentre eles, Alagoas, Bahia, Paraíba e Sergipe; na região Centro-Oeste, apenas o estado do Goiás; na região Sul, não houve estados que informaram a emissão; na região Sudeste, todos os estados informaram que emitem atestados e receitas por meio de teleconsultas (Figura 2).

Figura 2. Porcentagem dos estados do Brasil que informaram emitir receitas e atestados durante o atendimento por teleconsulta.

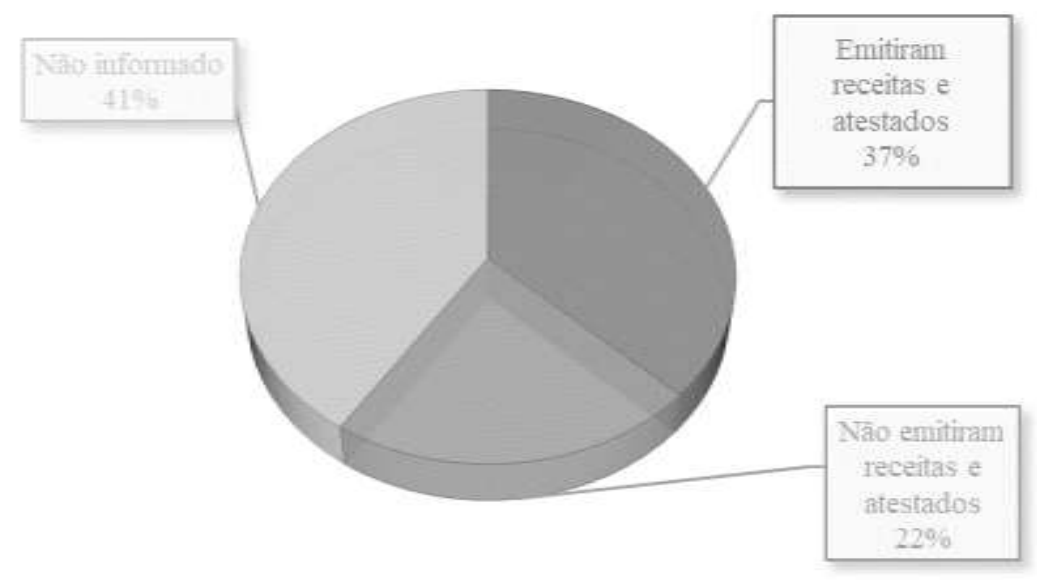

Fonte: Autores.

A porcentagem dos estados que divulgaram os serviços de teleconsulta durante a pandemia constituem um total de $77,8 \%$ dos estados. A mesma porcentagem (77,8\%) também se deu quanto a oferta de teleconsulta por ligação telefônica, constituindo como o principal meio de comunicação entre profissionais e usuários. A teleconsulta por videoconferência teve uma porcentagem de 55,6\%. A oferta de teleconsulta pelos estados utilizando outros instrumentos é de 70,4\%, dentre essas ferramentas temos um maior número de estados utilizando o WhatsApp, Gmail, Google Meet, Skype, Zoom, Chat Online, FaceTime, Hangout e seus próprios aplicativos. Quanto ao treinamento para utilizar as ferramentas, apenas 51,9\% dos estados ofertaram, dentre os estados que não informaram que dispõe, destaca-se: Alagoas, Bahia, Ceará, Distrito Federal, Espírito Santo, Maranhão, Pará, Rio de Janeiro, Rio Grande do Norte, Roraima, Sergipe, São Paulo e Tocantins (Figura 3). 
Figura 3. Porcentagem dos estados que divulgaram e promoveram os serviços de teleconsulta de forma acessível à população e aos profissionais da saúde.

Foi divulgada a oferta de teleconsulta

Foi feita teleconsul ta por ligação telefônica

Foi feita teleconsulta por videoconferência

Foi feita teleconsulta utilizando outras ferramentas

Os profissionais foram treinados para usar as ferramentas

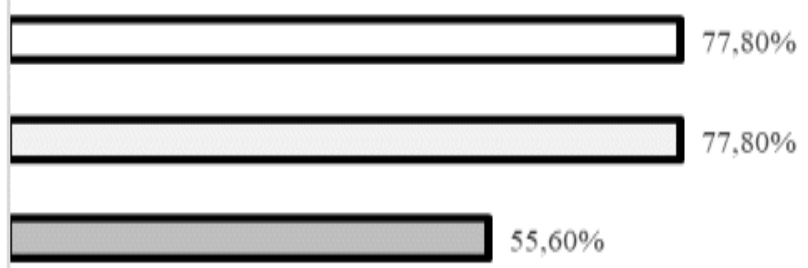

$70,40 \%$

$51,90 \%$

Fonte: Autores.

\subsection{Distribuição dos serviços de Telessaúde}

A distribuição da oferta dos serviços de telessaúde entre os estados difere, onde 85,2\% dispõem do programa Telessaúde. Os estados que não dispõem desse programa são Rondônia, Tocantins e o Distrito Federal, somente a Paraíba está em processo de planejamento para implantação. A teleconsultoria apresenta-se em $85,2 \%$ dos estados, os que não informaram ou não possuem são: Amapá, Tocantins, Rondônia e o Distrito Federal. A oferta de telediagnóstico está presente em 66,7\% dos estados, estando ausente ou não informado nos sites oficiais do Ceará, Maranhão, Rondônia, Sergipe, Amapá, Espírito Santo, Tocantins e no Distrito Federal. Quanto à oferta de teleducação, 63\% dos estados ofertam, os estados que não informaram ou não ofereceram são Amapá, Distrito Federal, Tocantins, Goiás, Mato Grosso, Mato Grosso do Sul, Pernambuco, Rio Grande do Sul e Rondônia. Os serviços de telerrastreamento são os menos oferecidos, constituindo 14,8\% dos estados, sendo os que ofertam Amazonas, Acre, Bahia e Pernambuco (Quadro 2).

Quadro 2. Distribuição da oferta dos serviços de Telessaúde entre os estados do Brasil.

\begin{tabular}{|c|c|c|c|c|c|c|}
\hline & Estados & $\begin{array}{c}\text { Dispõe do programa } \\
\text { Telessaúde }\end{array}$ & $\begin{array}{c}\text { Dispõe de } \\
\text { teleconsultoria }\end{array}$ & $\begin{array}{c}\text { Dispõe de } \\
\text { telediagnóstico }\end{array}$ & $\begin{array}{c}\text { Dispõe de } \\
\text { tele-educação }\end{array}$ & $\begin{array}{c}\text { Dispõe de } \\
\text { telerrastreamento }\end{array}$ \\
\hline \multirow{9}{*}{$\begin{array}{l}\mathbf{N} \\
\mathbf{O} \\
\mathbf{R} \\
\mathbf{D} \\
\mathbf{E} \\
\mathbf{S} \\
\mathbf{T} \\
\mathbf{E}\end{array}$} & Paraíba & $\begin{array}{c}\text { Em fase de } \\
\text { planejamento }\end{array}$ & $\begin{array}{c}\text { Em fase de } \\
\text { planejamento }\end{array}$ & $\begin{array}{l}\text { Em fase de } \\
\text { planejamento }\end{array}$ & $\begin{array}{c}\text { Em fase de } \\
\text { planejamento }\end{array}$ & $\begin{array}{c}\text { Em fase de } \\
\text { planejamento }\end{array}$ \\
\hline & Bahia & Sim & Sim & Sim & Sim & Sim \\
\hline & Sergipe & Sim & Sim & $\begin{array}{l}\text { Não ou Não } \\
\text { informado }\end{array}$ & $\begin{array}{l}\text { Não ou Não } \\
\text { informado }\end{array}$ & $\begin{array}{l}\text { Não ou Não } \\
\text { informado }\end{array}$ \\
\hline & Alagoas & Sim & Sim & Sim & Sim & $\begin{array}{l}\text { Não ou Não } \\
\text { informado }\end{array}$ \\
\hline & Pernambuco & Sim & Sim & Sim & Sim & Sim \\
\hline & $\begin{array}{l}\text { Rio Grande } \\
\text { do Norte }\end{array}$ & Sim & Sim & Sim & $\mathrm{Sim}$ & $\begin{array}{l}\text { Não ou Não } \\
\text { informado }\end{array}$ \\
\hline & Ceará & Sim & Sim & $\begin{array}{l}\text { Não ou Não } \\
\text { informado }\end{array}$ & $\begin{array}{l}\text { Não ou Não } \\
\text { informado }\end{array}$ & $\begin{array}{l}\text { Não ou Não } \\
\text { informado }\end{array}$ \\
\hline & Piauí & Sim & Sim & Sim & Sim & $\begin{array}{l}\text { Não ou Não } \\
\text { informado }\end{array}$ \\
\hline & Maranhão & Sim & Sim & $\begin{array}{l}\text { Não ou Não } \\
\text { informado }\end{array}$ & $\begin{array}{l}\text { Não ou Não } \\
\text { informado }\end{array}$ & $\begin{array}{l}\text { Não ou Não } \\
\text { informado }\end{array}$ \\
\hline \multirow{5}{*}{$\begin{array}{l}\mathbf{N} \\
\mathbf{O} \\
\mathbf{R} \\
\mathbf{T} \\
\mathbf{E}\end{array}$} & Amapá & $\begin{array}{l}\text { Não ou Não } \\
\text { informado }\end{array}$ & $\begin{array}{l}\text { Não ou Não } \\
\text { informado }\end{array}$ & $\begin{array}{l}\text { Não ou Não } \\
\text { informado }\end{array}$ & $\begin{array}{l}\text { Não ou Não } \\
\text { informado }\end{array}$ & $\begin{array}{l}\text { Não ou Não } \\
\text { informado }\end{array}$ \\
\hline & Amazonas & Sim & Sim & Sim & Sim & Sim \\
\hline & Acre & Sim & Sim & Sim & Sim & Sim \\
\hline & Rondônia & $\begin{array}{l}\text { Não ou Não } \\
\text { informado }\end{array}$ & $\begin{array}{l}\text { Não ou Não } \\
\text { informado }\end{array}$ & $\begin{array}{l}\text { Não ou Não } \\
\text { informado }\end{array}$ & $\begin{array}{l}\text { Não ou Não } \\
\text { informado }\end{array}$ & $\begin{array}{l}\text { Não ou Não } \\
\text { informado }\end{array}$ \\
\hline & Roraima & Sim & Sim & Sim & Sim & Não ou Não \\
\hline
\end{tabular}


Research, Society and Development, v. 10, n. 8, e54010817675, 2021

(CC BY 4.0) | ISSN 2525-3409 | DOI: http://dx.doi.org/10.33448/rsd-v10i8.17675

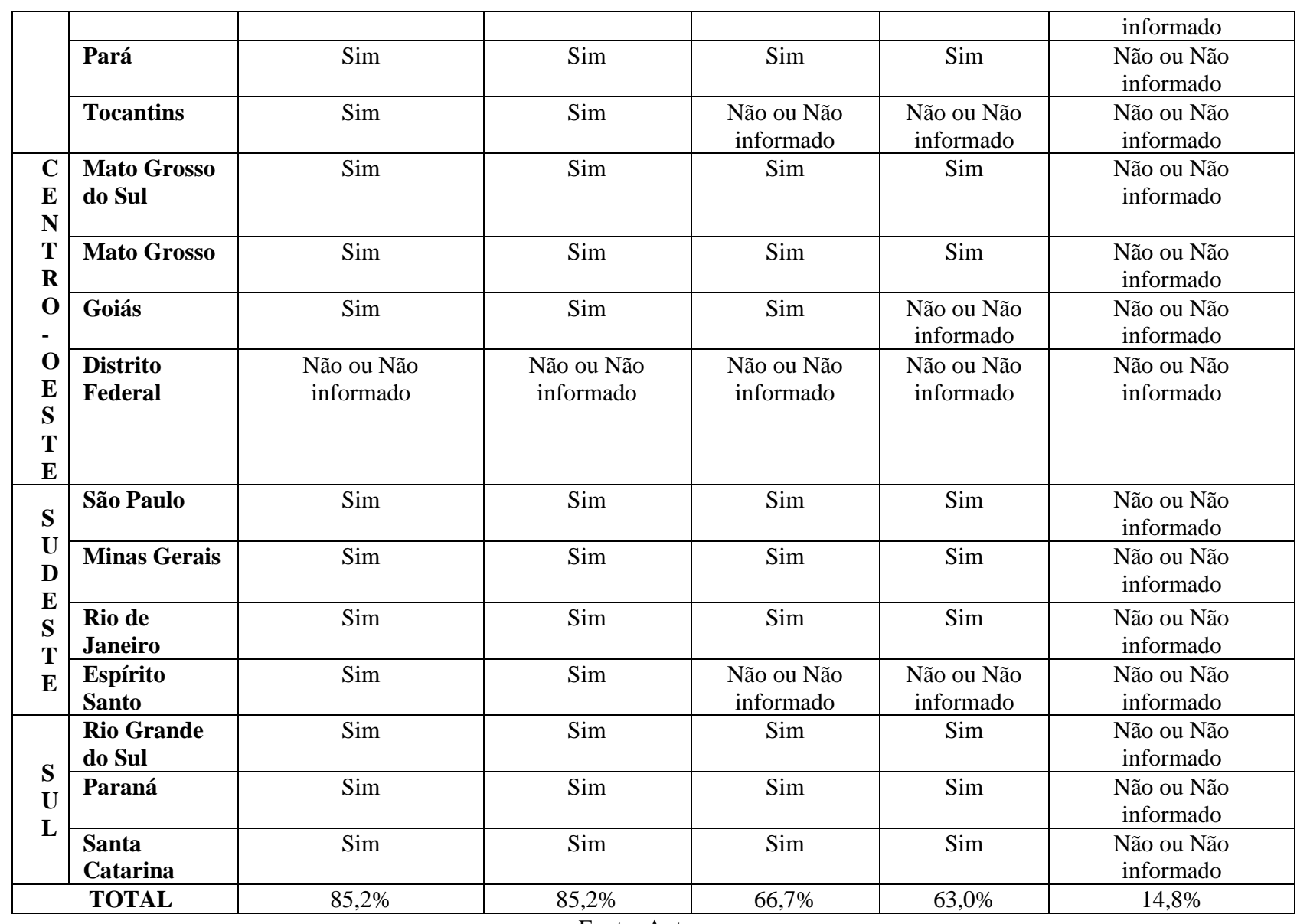

Fonte: Autores.

Em relação às características e funcionalidades da plataforma do Telessaúde, verifica-se que 57,1\% das plataformas demonstram ser acessíveis por dispositivo móvel, que 30\% das plataformas permitem anexar imagens e arquivos integrados ao SIGA/Saúde e ao CNES, e apenas 7,4\% delas enviam alertas e notificações por e-mails.

Quanto aos estados que lançaram suas próprias propostas, 74,1\% ofertaram teleconsulta durante a pandemia, 18,5\% dos estados não lançaram propostas e 7,4\% não informaram. Dessa forma, as principais propostas lançadas pelos estados foram, aplicativo "Coronavírus" (Amapá), aplicativo "Juntos no Combate ao Covid19" (Amazonas), disque "Coronavírus" (Acre), "Disque Corona" (Rondônia), aplicativo "Atende em casa" (Pará), aplicativo "Monitora Covid" (Paraíba), aplicativo "Atende em Casa" (Pernambuco), Teleatendimento Covid19 (Rio Grande do Norte), site COVID-19 | Coronavírus (Ceará), aplicativo "Monitora COVID-19" (Piauí), site "Triagem COVID-19" (Mato Grosso do Sul), aplicativo "Alô Saúde Floripa" (Santa Catarina), aplicativo "e-saúdesp" (São Paulo), aplicativo "Saúde Digital MG" (Minas Gerais) e aplicativo "Telessaúde Rio" (Rio de Janeiro). Enquanto outros estados não informaram propostas próprias. Vale ressaltar que esses aplicativos dispõem de diferentes funcionalidades de assistência à saúde, dentre elas, consultas pré-clínicas, teleconsultas e teleducação.

\section{Discussão}

A necessidade de conter a disseminação do Sars-Cov-2 impulsionou a adoção de medidas que priorizaram o atendimento apenas a nível de urgência e emergência, comprometendo aqueles classificados como eletivos. Além disso, houve limitação dos serviços disponibilizados nas Unidades Básicas de Saúde (UBS) e restrição do atendimento apenas para pessoas com sintomas de COVID-19 nas Unidades de Pronto Atendimento (Brasil, 2021). Desse modo, foi imprescindível recorrer ao teleatendimento em saúde para suprir a assistência que foi impossibilitada devido às medidas restritivas. De acordo com esta pesquisa, todos os estados brasileiros desenvolveram métodos para promover assistência pré-clínica a fim de auxiliar a 
compreender os sintomas da COVID-19. No entanto, no que se refere ao atendimento por teleconsulta, apenas a região CentroOeste e Sudeste ofereceram esse serviço para os cidadãos de todos os estados. Esses dados estão em consonância com o de outros trabalhos, que registraram diferenças no investimento prestado em Saúde Digital entre as diversas regiões do Brasil, além de significativas desigualdades na oferta da teleconsulta, principalmente devido às limitações na qualidade da conexão à Internet, falta de instruções quanto ao uso das ferramentas digitais e falta de interesse para implementação da teleconsulta na Unidade de Saúde da Família (Catapan, Willemann, \& Calvo, 2021).

Ao comparar os resultados obtidos nesta pesquisa com os dados descritos em outro estudo (Damasceno \& Caldeira, 2019), observa-se semelhança no que concerne aos serviços multiprofissionais ofertados por teleconsulta no SUS, sendo a maior parte realizado pela categoria dos enfermeiros, enquanto os cirurgiões-dentistas demonstram menor adesão ao modelo de teleconsulta. Um fator determinante para limitar a implementação dessa ferramenta é a falta de medidas que englobe e promova equidade durante o atendimento de pessoas com necessidades especiais (Piran et al., 2021). Segundo dados desta pesquisa, apenas alguns estados da região Centro-Oeste ofertaram recursos e desenvolveram projetos para atender usuários com necessidades especiais.

No Brasil, por meio da Medida Provisória N 2.220-2/01, foi instituída a Infraestrutura de Chaves Públicas Brasileira (ICP-Brasil), visando garantir a autenticidade, a integridade e a validade jurídica de documentos em forma eletrônica (Planalto, 2001). A partir dessa medida provisória e em decorrência da crise pandêmica causada pelo Sars-Cov-2, o Ministério da Saúde publicou a Portaria No 467/2020 que regulamenta, em caráter excepcional e temporário, as ações de telemedicina, assim como, contempla a emissão de receitas e atestados médicos à distância, desde que assinado com certificado digital no padrão de ICPBrasil, de forma gratuita (Brasil, 2020d). Desse modo, possibilitou que médicos e cirurgiões-dentistas emitissem atestados e receitas digitais, porém, com exceção da prescrição de medicamentos controlados, que necessitam de receita de controle especial situação que pode contribuir para justificar a baixa adesão dos estados brasileiros, tendo em vista que apenas $37 \%$ dos estados informaram emitir receitas e atestados de forma digital para os cidadãos na rede pública de saúde (Brasil, 2020b).

Os serviços de teleconsulta são ofertados na maioria das vezes por ligação telefônica, mas também são usados aplicativos de videoconferências ou outras ferramentas como WhatsApp, sendo a escolha pelo tipo de modalidade de atendimento condicionada à situação clínica e de acordo com a decisão do profissional e da pessoa a ser atendida (Harzheim \& D'Avila, 2020). Esses dados estão de acordo com o disponibilizado na literatura (Silva et al., 2021), na qual relatam o uso de equipamentos e ferramentas diversas para a teleconsulta, como: computador, notebook, tablet, telefone fixo, celular com acesso à Internet e citam o uso dos aplicativos Skype, Face Time, Hangout, Google Meet, plataformas institucionais, entre outras.

Sobre os serviços de Telessaúde Brasil Redes, observou-se que 85,2\% dos estados brasileiros oferecem esse recurso, sendo que a maioria dispõe de teleconsultoria e a minoria oferta telerrastreamento, o mesmo é observado por Belber e colaboradores (2021), nesse estudo eles ressaltam ainda a importância do Programa Telessaúde Brasil Redes para impedir a sobrecarga dos sistemas de saúde público, tendo em vista a possível resolutividade dos casos em nível de atenção básica, evitando que haja encaminhamento para outro serviço. Além disso, segundo Caetano e colaboradores (2020), o Telessaúde contribuiu para o enfretamento da COVID-19, e serviu como oportunidade para a consolidação da telemedicina no Brasil e de aperfeiçoamento do Sistema Único de Saúde. Pode-se afirmar que esse programa, em conjunto com as ferramentas digitais, oportunizou o surgimento da teleodontologia, possibilitando que os cuidados com a saúde bucal fossem retomados a partir da oferta de diversos serviços, como: telemonitoramento de casos clínicos, discussão de casos clínicos e teleconsultorias para os profissionais das UBS (Machado et al., 2021; Medeiros et al., 2020).

Alguns estados do Brasil $(74,1 \%)$ desenvolveram programas e aplicativos próprios, a fim de promover assistência aos cidadãos. Como o “Atende em casa - COVID-19”, lançado pela Prefeitura do Recife em conjunto com o Governo do Estado. 
Esses aplicativos têm o objetivo de evitar que a população procure as unidades de saúde desnecessariamente durante a situação de emergência causada pela pandemia da COVID-19, garantindo assim, orientações virtuais, que permitem uma classificação de risco do usuário e, se necessário, uma videochamada (teleorientação) com enfermeiros ou médicos (Binda \& Zaganelli, 2020).

Os resultados deste trabalho documental nas capitais brasileiras contribuem para a percepção de que a modalidade de assistência não presencial se tornou uma ferramenta de apoio significativa para os cidadãos e profissionais da saúde, desempenhando ações de teleatendimento de forma segura e rápida, devido à sua agilidade nos métodos de consultas e o incentivo das prefeituras e estados no desenvolvimento desses programas de saúde no período da pandemia da COVID-19.

O modelo de atendimento virtual irá se manter nos serviços de saúde, bem como em outros serviços, tanto na esfera pública quanto privada, devido aos benefícios percebidos ao longo dos atendimentos e acompanhamentos dos casos, principalmente na rede pública de saúde.

Esse trabalho demonstrou como os serviços de saúde e as capitais brasileiras se estruturaram para atender a população de modo remoto como resposta ao enfrentamento da COVID-19.

\section{Conclusão}

Os dados obtidos nessa pesquisa identificaram que a teleconsulta no SUS, seja ela por meio de aplicativos, mensagens ou ligações, possibilitou o acesso dos cidadãos aos serviços de saúde de forma remota, principalmente durante a pandemia da COVID-19. A maioria dos estados ofertou o Programa Telessaúde, dois estados e o Distrito Federal não disponibilizaram e um estado do Nordeste estava em processo de planejamento para a implementação do Programa.

No que tange às limitações do estudo, pode-se mencionar a indisponibilidade de algumas informações sobre a oferta de teleconsultas nos websites oficiais das Secretarias de Saúde em alguns estados do Brasil. Para futuras pesquisas, têm-se como proposta um estudo comparativo entre os resultados de consultas presenciais e a teleconsulta nos diagnósticos e tratamentos das doenças.

\section{Referências}

Almeida, S. G., Marabujo T., \& Gonçalves M. C. (2021). Grado de satisfacción de los pacientes de la Unidad de Salud Familiar Vitrius con la teleconsulta durante la pandemia del COVID-19. Semergen, 47(4): 248-255.

Belber, G. S., dos Santos Passos, V. C., da Costa Borysow, I., \& Maeyama, M. A. (2021). Contribuições do Programa Nacional Telessaúde Brasil Redes na formação de recursos humanos na atenção básica. Brazilian Journal of Development, 7(1): 1198-1219.

Bergman, J., Ballin, M., Nordström, A. \& Nordström, P. (2021). Risk factors for COVID-19 diagnosis, hospitalization, and subsequent all-cause mortality in Sweden: a nationwide study. Eur J Epidemiol, 36: 287-298.

Brasil. Atenção Primária em Saúde (2007). O Programa Nacional Telessaúde Brasil Redes foi instituído por meio da Portaria do Ministério da Saúde no 35 de janeiro de 2007, e redefinido e ampliado por meio da Portaria MS n ${ }^{\circ} 2.546$, publicada no dia 27 de outubro 2011 . https://aps.bvs.br/programa-nacionaltelessaude-brasil-redes/

Binda Filho, D. L., \& Zaganelli, M. V. (2020). Telemedicina em Tempos de Pandemia: Serviços Remotos de Atenção à Saúde no Contexto da COVID-19. Humanidades e Tecnologia (FINOM), 25(1): 115-133.

Brasil. Lei N ${ }^{\circ}$ 13.989. DE 15 De Abril De 2020. (2020a). Dispõe sobre o uso da telemedicina durante a crise causada pelo coronavírus (SARS-CoV-2). https://www.in.gov.br/en/web/dou/-/lei-n-13.989-de-15-de-abril-de-2020-252726328

Brasil. Medida Provisória No 2.200-2, DE 24 DE AGOSTO DE 2001 (2001). Institui a Infra-Estrutura de Chaves Públicas Brasileira - ICP-Brasil, transforma o Instituto Nacional de Tecnologia da Informação em autarquia, e dá outras providências. Diário Oficial da União. http://www.planalto.gov.br/ccivil_03/mpv/antigas_2001/2200-2.htm

Brasil. Ministério da Saúde (2021). Guia de orientações para atenção odontológica no contexto da COVID-19. https://www.gov.br/saude/ptbr/media/pdf/2020/novembro/17/17_12_guia-de-orientacaoes-para-atencao-odontologica-no-contexto-da-covid-19.pdf

Brasil. Ministério da Saúde. (2020b). Secretaria de Atenção Primária à Saúde (SAPS). SUS terá Consultório Virtual da Saúde da Família. Portal do Ministério da Saúde. https://aps.saude.gov.br/noticia/8136. 
Brasil. Ministério da Saúde. (2020c). Secretaria de Atenção Primária à Saúde (SAPS). TeleSUS. Portal do Ministério da Saúde. https://aps.saude.gov.br/ape/corona/telesus.

Brasil. Portaria N 467, De 20 De Março De 2020 (2020d). Dispõe, em caráter excepcional e temporário, sobre as ações de Telemedicina, com o objetivo de regulamentar e operacionalizar as medidas de enfrentamento da emergência de saúde pública de importância internacional previstas no art. $3^{\circ}$ da Lei $\mathrm{n}^{\circ} 13.979$, de 6 de fevereiro de 2020, decorrente da epidemia de COVID-19. Diário Oficial da União 2020.http://www.planalto.gov.br/CCIVIL_03/Portaria/PRT/Portaria\%20n\%C2\%BA\%20467-20-ms.htm

Brasil. Resolução No 510, de 07 de abril de 2016. (2016). Conselho Nacional de Saúde, Instrução Normativa $n^{\circ}$ 510/2016, que dispõe sobre as normas aplicáveis a pesquisas em Ciências Humanas e Sociais. Diário Oficial da União. https://www.in.gov.br/materia//asset_publisher/Kujrw0TZC2Mb/content/id/22917581.

Caetano, R. et al. (2020). Desafios e oportunidades para telessaúde em tempos da pandemia pela COVID-19: uma reflexão sobre os espaços e iniciativas no contexto brasileiro. Cadernos de Saúde Pública, 36, e00088920.

Caetano, R., Silva, A. B., Guedes, A., Paiva, C., Ribeiro, G., Santos, D. L., \& Silva, R. (2020). Challenges and opportunities for telehealth during the COVID19 pandemic: ideas on spaces and initiatives in the Brazilian context. Desafios e oportunidades para telessaúde em tempos da pandemia pela COVID-19: uma reflexão sobre os espaços e iniciativas no contexto brasileiro. Cadernos de saúde pública, 36(5): e00088920.

Caram, S. C., Ramos, S. R. F., Almeida, G. N. \& Brito, M. J. M. (2021). Sofrimento moral em profissionais de saúde: retrato do ambiente de trabalho em tempos de COVID-19. Revista Brasileira de Enfermagem, 74: e20200653.

Catapan, S. D. C., Willemann, M. C. A., \& Calvo, M. C. M. (2021). Estrutura e processo de trabalho para implantação da teleconsulta médica no Sistema Único de Saúde do Brasil, um estudo transversal com dados de 2017-2018. Epidemiologia e Serviços de Saúde, 30 , e2020305.

Damasceno R. F., \& Caldeira A. P. (2019). Fatores associados à não utilização da teleconsultoria por médicos da Estratégia Saúde da Família. Ciência e Saúde Coletiva, 24(8): 3089-3098.

Domingues, S. A. M. C. (2021). Desafios para a realização da campanha de vacinação contra a COVID-19 no Brasil. Cadernos de Saúde Pública, 37 (1): e00344620.

Ghiglia, C. M. M. (2020). Telemedicina: su rol en las organizaciones de salud. Revista Médica del Uruguay, 36(4): $411-417$.

Harzheim, E. et al. (2020). Ações federais para apoio e fortalecimento local no combate ao COVID-19: a Atenção Primária à Saúde (APS) no assento do condutor. Ciência \& Saúde Coletiva, 25, 2493-2497.

Lakatos, E. M. Marconi M. A. (2009). Técnica de pesquisa. (6a ed). Atlas.

Machado, F. C., Oliveira, L. C., Silva, D. L. M., Carvalho, T. A., Novais, V. R., \& Menezes, M. S. (2021). Teleorientação com o uso de ferramentas digitais no auxílio ao atendimento odontológico em época de pandemia da COVID-19: revisão integrativa da literatura. Research, Society and Development, 10(6): e17210615663.

Medeiros, A. M. B et al. (2020). Os desafios da telemedicina frente a relação médico -pacienteem tempos de Covid -19. Research, Society and Development, 9(10): e9219109379.

Nazif-Muñoz, I. J., Peña, S. \& Oulhote, Y. (2021). The global viralization of policies to contain the spreading of the COVID-19 pandemic: Analyses of school closures and first reported cases. Plos One, 16(4): e 0248828

O'Keefe, B. J., Tong, J. E., O'Keefe. D. G. \& Tong, C. D. (2021). Description of symptom course in a telemedicine monitoring clinic for acute symptomatic COVID-19: a retrospective cohort study. BMJ Open, 11(3): e044154.

Piran C. M. G. et al. (2021) Atendimento à pessoas com deficiência na atenção primária à saúde em tempos de Covid-19. Revista Brasileira de Crescimento e Desenvolvimento Humano, 7(5): 45039-45049.

Sá-Silva, J. R., Almeida, C. D. \& Guindani, J. F. (2009). Pesquisa documental: pistas teóricas e metodológicas. Revista Brasileira de História \& Ciências Sociais, 1: 2175-3423.

Villela, M. F. E. et al. (2021). COVID-19 outbreak in Brazil: adherence to national preventive measures and impact on people's lives, an online survey. BMC Public Health, 152(21): 1-10. 